\title{
Simulation of Acquisition Artefacts in MR Scans: Effects on Automatic Measures of Brain Atrophy
}

\author{
Oscar Camara-Rey ${ }^{1}$, Beatrix I. Sneller ${ }^{1}$,Gerard R. Ridgway ${ }^{1}$, Ellen Garde $^{2}$, \\ Nick C. Fox ${ }^{2}$, and Derek L. G. Hill ${ }^{1}$ \\ ${ }^{1}$ Center of Medical Image Computing, University College of London, UK \\ o.camara-rey@ucl.ac.uk \\ ${ }^{2}$ Dementia Research Centre, Institute of Neurology, \\ University College Of London, UK
}

\begin{abstract}
Automatic algorithms in conjunction with longitudinal MR brain images can be used to measure cerebral atrophy, which is particularly pronounced in several types of dementia. An atrophy simulation technique has been devised to facilitate validation of these algorithms. To make this model of atrophy more realistic we simulate acquisition artefacts which are common problems in dementia imaging: motion (both step and periodic motion) and pulsatile flow artefact. Artefacts were simulated by combining different portions of k-space from various modified image. The original images were 7 MR scans of healthy elderly controls, each of which had two levels of simulated atrophy. We investigate the effect of the simulated acquisition artefacts in atrophy measurements provided by an automatic technique, SIENA.
\end{abstract}

\section{Introduction}

Structural magnetic resonance imaging (MRI) can be used to estimate the progression of neurodegenerative disease since brain atrophy is a measurable feature of the most common forms of dementia such as Alzheimer's disease (AD). Several computerized techniques [1-4] for the quantification of cerebral atrophy in MR scans are available in the literature. Nevertheless, there is a lack of consensus on the most appropriate method to be used since standardized test datasets with known atrophy rates are not available.

We have described [5] a method based on the combination of expert knowledge of clinical changes in brain with finite-element methods, providing valuable groundtruth data for the objective validation of atrophy measurement techniques. This technique, however, did not take into account the common presence of artefacts in the MR scans due to imperfections in the acquisition, which may have a strong impact on the performance of the subsequent atrophy quantification techniques. For instance, movement during the acquisition of the edge of $\mathrm{k}$ space can lead to a ringing artefact from the scalp [6], global movement during the central portion of k-space would 
generate obvious ghosts and blur image intensities, or flow artefacts may appear in the medial temporal lobes due to the pulsatile blood flow in the carotid arteries. All these artefacts have the potential to severely degrade quantitative MR-based analysis such as measurement of atrophy.

The main goal of this paper is to present the techniques we are developing to simulate acquisition artefacts in order to make the generated ground-truth data more realistic. Furthermore, these simulations will allow the assessment of post-processing artefact correction techniques $[6,7,8]$ and their incorporation in atrophy measurement methods.

One of the most common causes of acquisition artefacts is subject motion. Rotational motion artefacts were simulated by Blumenthal et al. [9] to study their effects on a GM quantification technique and by Preboske et al. [10] to investigate their impact on a semi-automatic atrophy measurement method, the boundary shift integral [1]. Nevertheless, in some particular brain regions, other factors can be as important as subject motion. For instance, the main cause of image degradation in the temporal lobe, a region with a crucial role in Alzheimer's disease, is pulsatile flow artefact from carotid arteries. In clinical studies related to dementia, out of 837 subjects, we have visually identified 105 (12.5\%) of the subjects' images with artefacts located in the temporal lobes, which can be classified according to their cause: $68(64.8 \%)$ with flow artefact; $16(15.2 \%)$ with motion-related artefact; 4 $(3.8 \%)$ with susceptibility artefacts; and $17(16.2 \%)$ images with artefacts induced by other factors.

Therefore, we have developed techniques to simulate the two most significant causes of acquisition artefacts, a rotational motion during the scan, and a pulsatile motion. We have then applied SIENA [2], an automatic technique for the estimation of atrophy rates, to different sets of atrophy simulated data with the presence of acquisition artefacts.

\section{Method}

The generation of ground-truth data from an MR scan for the evaluation of atrophy measurement techniques can be divided in two stages: simulation of atrophy and simulation of acquisition artefacts.

\subsection{Simulation of Atrophy}

The method for atrophy simulation can be divided into four phases: meshing of a labelled brain atlas; adaptation of the generated mesh to an individual MR scan; introduction of the individual-specific mesh into a FEM solver that will generate the simulated deformations; and the application of such deformations to the MR scan. More details about this method can be found in [5]. Figure 1 shows an example of atrophy simulation. 

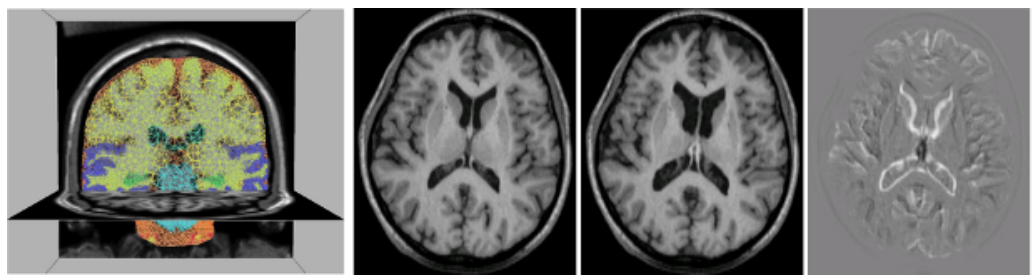

Fig. 1. From left to right: mesh, baseline, atrophy simulated scan and difference image

\subsection{Simulation of Acquisition Artefacts}

\subsubsection{Rotational Motion}

Rotational motion during a scan causes inconsistencies in the sampled k-space signal that result in blurring and ghosting in the image domain. The method we developed to simulate rotational motion is the following.

A motion-free 3D image volume (top left of Fig. 2) is converted into a complex image by treating the modulus image as the real part, and setting the imaginary component to zero. This complex image is then rotated by 5 degrees about an axis through the internal auditory meatus, simulating a slight nodding of the patient during the scan (bottom left of Fig. 2). Bicubic interpolation was used in the rotation, and voxels entering the volume were assigned the value zero. The original and rotated images were then both Fourier transformed into k-space (second column of Fig. 2). A motion-corrupted k-space was subsequently generated by assembling portions of the k-spaces corresponding to the un-rotated and rotated images (third column of Fig. 2). The motion-corrupted k-space was then transformed back to the image domain (final image of Fig. 2) to give the motion corrupted volume. The k-space samples were

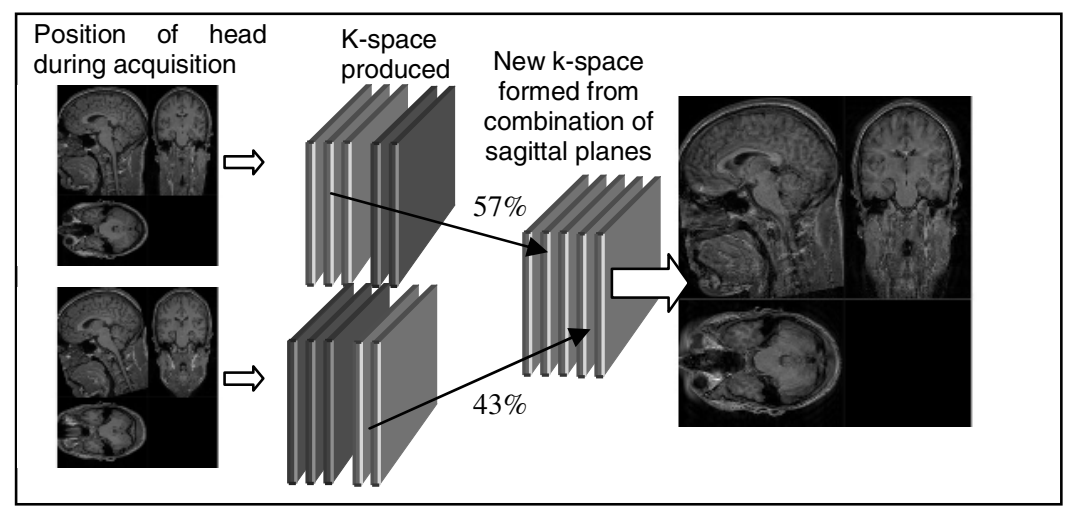

Fig. 2. Simulation of step rotational motion: motion is applied in the spatial domain to a motion-free image volume, and the k-spaces re-combined to generate a motion-corrupted image volume 
combined as sagittal planes representing a sagittal MR acquisition orientation. Transverse or coronal planes could also have been simulated in these 3D volumes by appropriately assembling the final $\mathrm{k}$-space.

The appearance of the motion artefacts is influenced by which region (its distance to the centre of the k-space) of k-space is being sampled as the motion takes place, and whether there is a single step motion, or a periodic motion. Our technique allows complete freedom in the selection of the time of the motion in the scan, and for the examples in this paper, we simulated step motion by combining the first $57 \%$ of the un-rotated k-space, with $43 \%$ of the rotated k-space. We simulated the motion just after half way through the acquisition as the artefact is more noticeable the nearer to the centre of k-space it occurs. Periodic motion, representing a repeated nodding of the head, was simulated by generating the motion-corrupted k-space from portions of the un-rotated and rotated $\mathrm{k}$-space, alternating between the two at $44 \%, 48 \%, 52 \%$ and 56\%. Figure 3 shows an example of the images resulting from simulated step and periodic rotational motion.
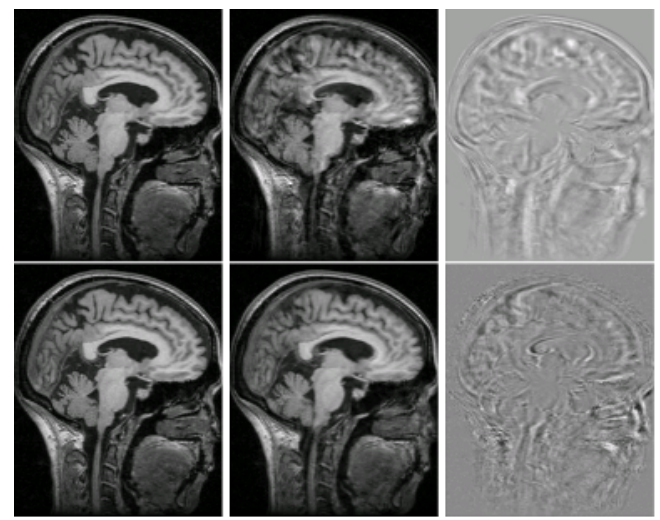

Fig. 3. Top: periodic motion, bottom: step motion. From left to right: Atrophy simulated image, atrophy plus motion artefact simulated images, difference image.

\subsubsection{Pulsatile Motion}

Another important artefact in MR images is pulsatile motion, which typically leads to streaks across the image in the slow-phase encoding direction. These artefacts arise from major blood vessels in the field of view, such as the carotid artery where it passes through the carotid siphon, and result from a signal in the vessel that varies from shot to shot during the acquisition. Simulated pulsatile motion artefacts were added to the simulated data as follows (see also Figure 4).

The 3D modulus images were converted to a complex image as described above, and four different coil views were obtained by multiplying the image by four separate coil sensitivity profiles. Because the pulsatility artefact is predominantly in the 
slow- phase encode direction, each plane of k- space was treated as being acquired instantaneously (contrast being largely determined by the acquisition of the centre portion of k-space) and $1.73 \mathrm{~s}$ apart from the next. A cylinder corresponding to the carotid artery was manually delineated from the image, and the intensity of this modulated according to a literature-based model of the flow in the carotid artery [11]. For each shot in the slow-phase encode direction, a different image was simulated by modulating the intensity of the carotid signal in the delineated cylinder. These images were transformed into the $\mathrm{k}$-space, and one $\mathrm{k}$-space plane from each image was combined to form a pulsatile-motion corrupted $\mathrm{k}$-space based on an estimated heart rate of $60 \mathrm{bpm}$. Because this is a one dimensional motion, it was implemented just for the lines containing the carotid, rather than the entire image, in order to reduce computational cost.

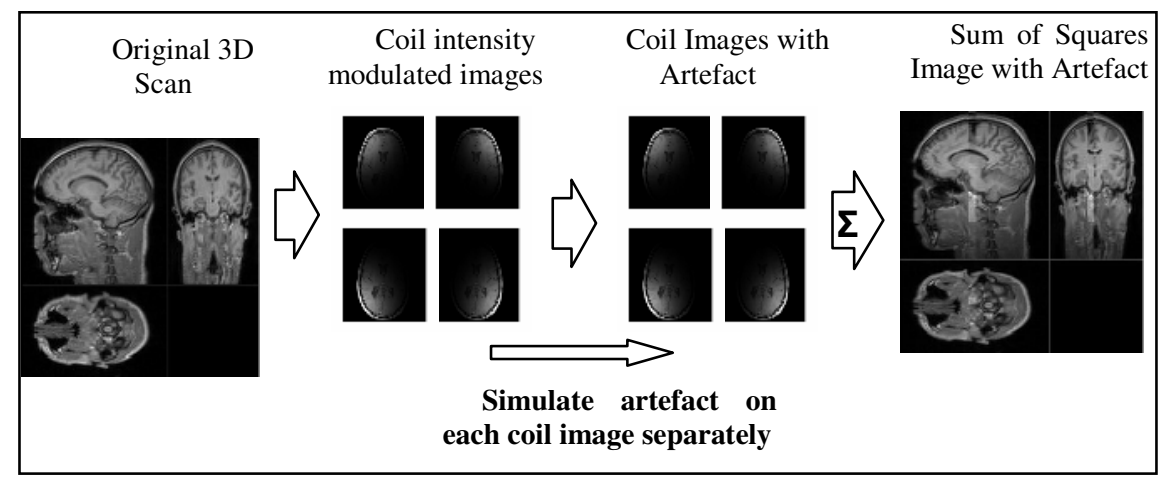

Fig. 4. Overview of simulation of flow artefact

The resulting image, as can be seen in Figure 5 has a simulated flow artefact running right to left. This is because we simulated the slow phase encode direction in the left-right direction as is common in $3 \mathrm{D}$ volumes used in dementia imaging. This is implemented in the simulation by using sagittal planes of k-space. However we could simulate any direction of acquisition which would alter the direction of the simulated artefact, and could also simulate more complex vessel geometries or flow properties.
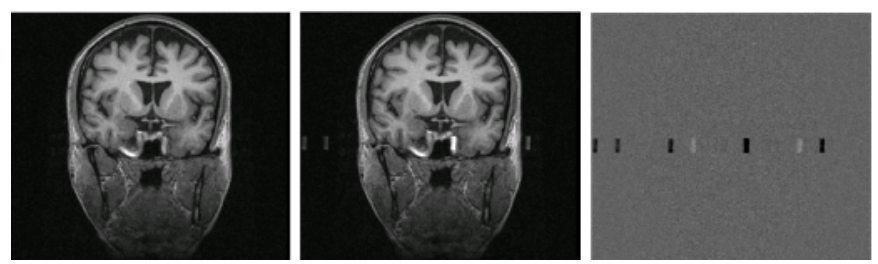

Fig. 5. From left to right: Atrophy simulated image; atrophy and flow artefact simulated image; difference image 


\section{Experiment}

\subsection{Design}

We have designed an experiment in order to investigate the effect of the simulated acquisition artefacts in atrophy measurements provided by an automatic technique, SIENA (Structural Image Evaluation, using Normalization, of Atrophy), proposed by Smith et al. [2].

Firstly, this method segments brain from non-brain tissue in the head, and estimates the outer skull surface. Then, it uses these results to register the two brains with the skulls constrained. These images are then segmented, and the atrophy is estimated from the movement of the segmentation boundaries. The output is the estimation of the percentage of brain volume change between the two scans, as well as a flow image in which regions of local change are shown, as can be seen in Figure 5. The authors of this technique claim a median absolute error of $0.2 \%$ of the whole brain volume change in a validation study.

In this work, we have employed 7 MR scans of healthy elderly controls enrolled in a longitudinal research project at the anonymous Centre, anonymous Institute, anonymous University, anonymous country and underwent serial MRI, clinical and neuropsychological assessment over a period of at least one year. T1-weighted volumetric MR images were acquired on a 1.5 Tesla Signa Unit (General Electric, Milwaukee) using a $256 * 256$ matrix to provide 124 contiguous $1.5 \mathrm{~mm}$ coronal slices through the head (acquisition parameters: time to repeat, $15 \mathrm{~ms}$; time to echo, $5.4 \mathrm{~ms}$; flip angle $15^{\circ}$, field of view $24 * 24 \mathrm{~cm}$ ).

We have applied the atrophy simulation technique [6] to these 7 MR scans, generating two new images, Atsim1 and Atsim2, for each subject scan (baseline) with different amounts of regional and global atrophy in several brain tissue compartments and structures, resulting in a dataset of 21 images. In this work, we only make use of the atrophy simulation's ground truth values for the whole brain volume change since SIENA only gives an estimation of the global atrophy rate. The mean and standard deviation of the simulated whole brain volume change percentages are the following: a reduction of $5.11 \% \pm 0.23$ for Atsiml; and a reduction of $10.84 \% \pm 0.47$ for Atsim 2 .

The next phase of the experiment applies the methods described in Section 2 to the dataset of atrophy simulated images in order to simulate the acquisition artefacts detailed above. Specifically, we simulate step and periodic rotational motion (see Section 2.1) for the 21 available images and pulsatile motion (see Section 2.2) for a subset of 7 images (three different cases and their corresponding atrophy simulated images), due to the time-consuming manual segmentation of the carotid artery needed to simulate this artefact. Furthermore, we have obtained, for a single case and its associated Atsim 1 and Atsim 2 images, 10 different step and periodic rotational motion simulated images varying the severity of the artefact (i.e. changing degrees of rotation).

Finally, we run SIENA on the whole dataset of simulated images, aiming at studying the effect of acquisition artefacts on atrophy measurements. 


\subsection{Results}

Results are summarized in Table 1. We have computed the difference (diff1) between the whole brain atrophy measurement calculated by SIENA and the gold standard, for the images that contain both simulated atrophy and simulated artefacts. We have also computed the difference (diff2) in SIENA results obtained with just simulated atrophy, and with simulated atrophy plus simulated artefact. The main difference between both measures is that "diff1" accumulates SIENA errors in the estimation of atrophy as well as errors induced by the simulation of acquisition artefacts, while "diff2" is independent of the atrophy simulation stage.

Table 1. Effect of simulated acquisition artefacts. diff1: difference between SIENA results and the gold standard for atrophy and artefact simulated images. diff2: difference in SIENA results between just simulated atrophy and with simulated atrophy plus simulated artefact. diff1 and diff 2 are percentages of whole brain volume change.

\begin{tabular}{|c|c|c|c|c|}
\cline { 2 - 5 } \multicolumn{1}{c|}{} & No artefact & Periodic & Step & Flow \\
\hline diff1 & $0.286 \pm 0.383$ & $1.197 \pm 0.777$ & $1.123 \pm 0.904$ & $0.412 \pm 0.220$ \\
\hline diff2 & n/a & $0.982 \pm 0.659$ & $0.941 \pm 0.738$ & $0.479 \pm 0.099$ \\
\hline
\end{tabular}

Both types of difference measures suggest that the presence of artefacts in the images has a substantial impact on atrophy measurements obtained with SIENA. We can observe in Table 1 that rotational motion artefacts, either step or periodic, degrade the performance of SIENA more than flow artefacts. This is due to the limited region where pulsatile artefacts are present, mostly the temporal lobes, while SIENA only provides a global estimation of the whole brain volume change. It must be pointed out that the accuracy obtained with SIENA in atrophy measurements without artefacts (first column of Table 1) are consistent with results provided by the authors of this technique (accuracy of $0.2 \%$ of whole brain volume change).

Figure 6 shows the impact on the error in the SIENA atrophy measurement as a function of magnitude of the rotational motion (stop motion from $0^{\circ}$ to $10^{\circ}$ degrees in intervals of 1 degree). There is a strong correlation between the increase in atrophy measurement errors and the severity of the simulated artefact. The expected difference in whole brain volume change between $\mathrm{AD}$ and normal aging over a 12 month period is around $1.5 \%$. Results in Figure 6 suggest that rotational motions larger than 5 degrees of rotation could induce artefacts around the same order of these expected differences.

\section{Conclusions}

We have presented the techniques we have developed to simulate the two most common acquisition artefacts in MR scans, rotational (both step and periodic) and pulsatile motion, in order to make ground truth data for atrophy measurement techniques more realistic. The methodology can be used to simulate many different 


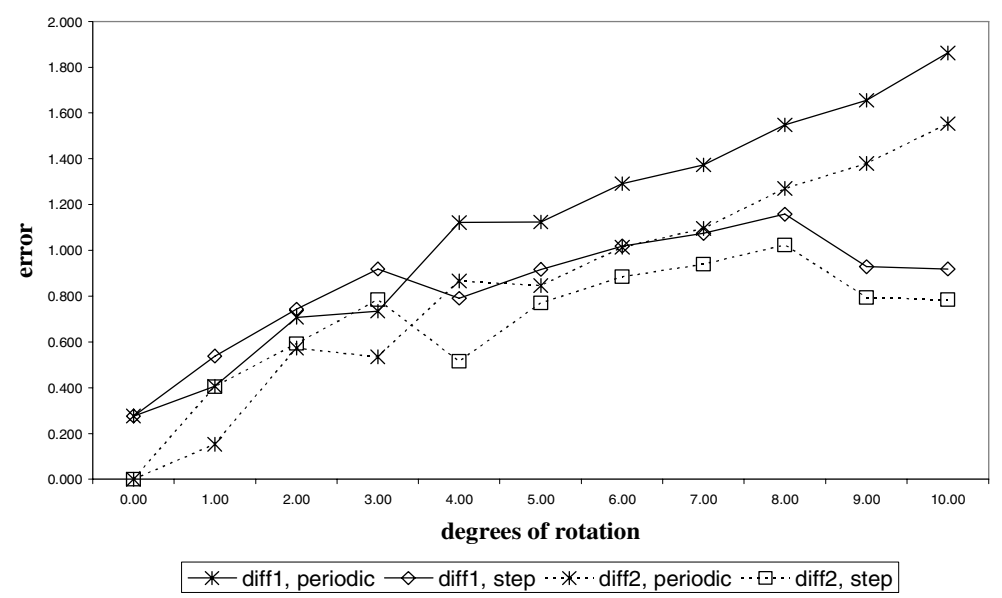

Fig. 6. Effect on SIENA results varying the severity of the rotational simulated artefacts

magnitudes of artefacts, and can be applied to many different atrophy quantification methods.

Only one atrophy quantification method has been investigated. The impact is likely to be different with different algorithms, and especially for local atrophy measures (e.g.: from a non-rigid registration algorithm) or cortical thickness measurement methods. The value of the approach is in predicting the sensitivity of methods to motion artefact (potentially valuable in a power analysis used in the design of a clinical trial), and also in comparing the relative merits of alternative atrophy measuring algorithms in the presence of common image artefacts.

\section{References}

1. Freeborough, P., Fox, N.: The boundary shift integral: an accurate and robust measure of cerebral volume changes from registered repeat MRI. IEEE Transactions on Medical Imaging 16 (1997) 623-629

2. Smith, S., Stefano, N.D., Jenkinson, M., Matthews, P.: Normalized accurate measurement of longitudinal brain change. Journal of Computer Assisted Tomography 25 (2001) 466-475

3. Davatzikos, C., Genc, A., Xu, D., Resnick, S.: Voxel-based Morphometry using the RAVENS maps: methods and validation using simulated longitudinal atrophy. Neuroimage 14 (2001) 1361-1369

4. Freeborough, P., Fox, N., Kitney, R.: Modeling brain deformations in Alzheimer's disease by fluid registration of serial 3D MR Images. Journal of Computer Assisted Tomography 22 (1998) 838-843

5. Camara, O., Schweiger, M., Scahill, R.I., Crum, W.R., Sneller, B.I., Schnabel, J.A, Ridgway, G.R., Cash, D.M., Hill, D.L.G., Fox N.C.: Phenomenological model of diffuse global and regional atrophy using Finite-Element methods. IEEE Transactions on Medical Imaging (2006). In press. 
6. Howarth, C., Hutton, C., Deichmann, R.: Improvement of the image quality of T1weighted anatomical brain scans. Neuroimage 29 (2005) 930-937

7. Atkinson, D., Hill, D.L., Stoyle, P.N., Summers, P.E., Clare, S., Bowtell, R., Keevil, S.F.: Automatic compensation of motion artifacts in MRI. Magnetic Resonance Medicine 41 (1999) 163-170

8. Manduca, A., McGee, K.P., Welch, E.B., Felmlee, J.P., Grimm, R.C., Ehman, R.L.: Autocorrection in MR imaging: adaptive motion correction without navigator echoes. Radiology 215 (2000) 904-909

9. Blumenthal, J.D., Zijdenbos, Al, Molloy, E., Giedd, J.N.: Motion artifact in magnetic resonance imaging: implications for automated analysis. Neuroimage 16 (2002) 89-92

10. Preboske, G.M., Gunter, J.L., Ward, C.P., Jack Jr., C.R.: Common MRI acquisition nonidealities significantly impact the output of the boundary shift integral method of measuring brain atrophy on serial MRI. Neuroimage (2006) In press.

11. Holdsworth, D., Norley, C., Frayne, R., Steinman, D., Rutt, B.: Characterization of common carotid artery blood-flow waveforms in normal human subjects. Physiological Measurement 20 (1999) 219-240 